\title{
Mapping the ionic fingerprints of molecular monolayers
}

Joshua Lehr', Justin R. Weeks', Adriano Santos², Gustavo T. Feliciano², Melany I. G. Nicholson'2, Jason J. Davis $^{1 \#}$ and Paulo R. Bueno ${ }^{2 *}$

${ }^{1}$ Department of Chemistry, University of Oxford, South Parks Road, Oxford OX1 3QZ, UK.

${ }^{2}$ Institute of Chemistry, Physical Chemistry Department, Univ. Estadual Paulista (São Paulo State University, UNESP), CP 355, 14800-900, Araraquara, São Paulo, Brazil.

\section{ABSTRACT}

We have previously proposed, and experimentally resolved, an ionic charge relaxation model for redox inactive self-assembled monolayers (SAMs) on metallic electrodes in contact with a liquid electrolyte. Here we analyse, by capacitance spectroscopy, the resistance and capacitance terms presented by a range of thiolated molecular films. Molecular dynamics simulations support a SAM-specific energy barrier to solution-phase ions. Once surmounted, the entrapped ions support a film embedded ionic capacitance and non-faradaic relaxation, which can be assigned as a particular case of general electrochemical capacitance.

Keywords: Self-assembled monolayer (SAM); capacitance spectroscopy (CS); molecular dynamic simulation; dipolar relaxation, dielectric relaxation in molecular/metallic interfaces; Cole-Cole relaxation.

\section{Corresponding authors}

Phone: +55 163301 9642; Fax: +55 1633222308

Email:*prbueno@iq.unesp.br; \#iason.davis@chem.ox.ac.uk 


\section{INTRODUCTION}

The preparation of well-defined organic self-assembled monolayers (SAMs) on a solid surface remains intensely important, ${ }^{1}$ because of its underpinning role in manipulating interfacial properties, and generating derived sensors ${ }^{2-6}$ and electronic junctions..$^{7-9}$ Many SAM-based applications are electrochemical in nature and are accordingly sensitive to structural, dynamic and electronic changes within the films, ${ }^{8,10-20}$ as well as to the effects of ionic ingress. ${ }^{21-25} \mathrm{We}^{26-29}$ and others ${ }^{21-25,}$, 30-35 have investigated the electrochemical and dielectric properties of these interfaces by employing electrochemical impedance spectroscopy (EIS) and capacitance spectroscopy (CS), the latter an impedance-derived immittance approach ${ }^{10}$ which resolves the timedependent responses both within the organized organic components and solution phase electrolyte. ${ }^{17,}{ }^{36}$ Standard EIS methods have been shown to have value in characterizing the electronic, ${ }^{1,37,38}$ ionic $^{23,25,31}$ steric $^{2}$ blocking effects of these insulating layers using a redox probe in solution, where both the current (in classical cyclic voltammetry) and charge transfer resistance $\left(R_{c t}\right.$, in impedimetric analysis) are responsive (usually, a concomitant decrease in current and an increase in $R_{c t}$ are observed from bare electrode to SAM modified electrode. For example, please see SI). ${ }^{39}$

In redox-active films, CS analysis is particularly useful in separating the faradaic and non-faradaic relaxations, as shown in Figure 1. Herein we focus the latter as typified by features presented by non-electroactive SAMs.
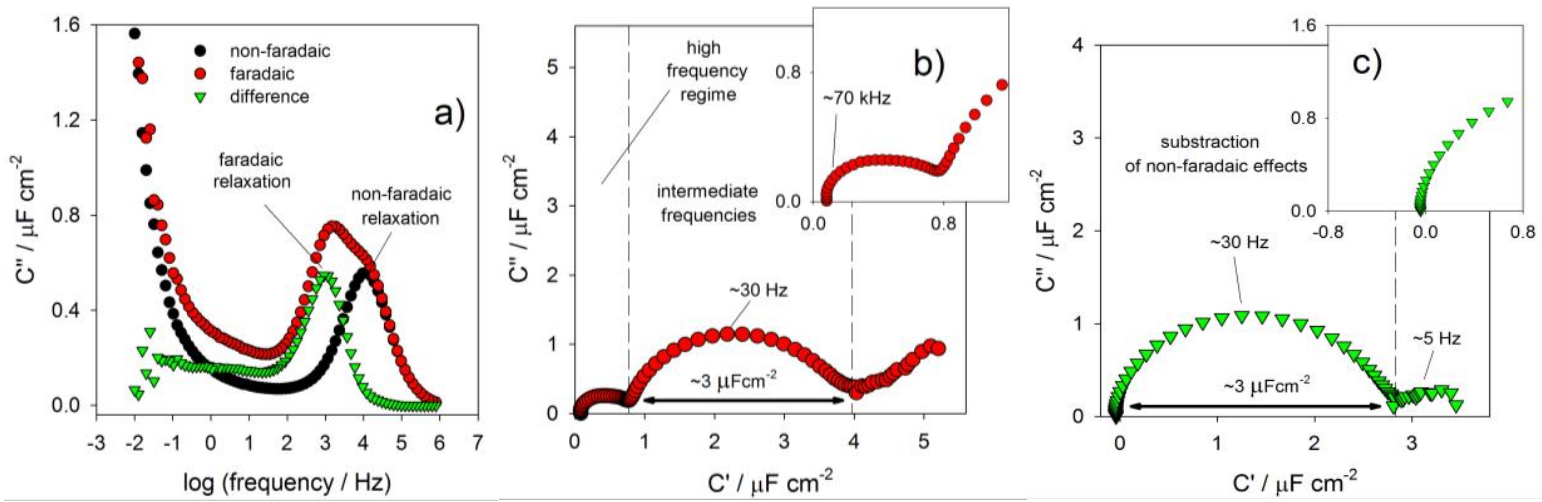

Figure 1. A clear separation of faradaic and non-faradaic capacitive responses in electro-active monolayers is possible by using CS. (a) and (b) are Bode and Nyquist capacitive diagrams. Non-faradaic contributions (black) are obtained outside of the redox active potential window. At appropriate surface potentials both faradaic and non-faradaic contributions are apparent (red). It is possible to subtract the latter in obtaining a "purified" faradaic response (green curve). (c) The resultant Nyquist plot, containing only faradaic contributions. Reprinted (adapted) with permission from (Paulo R. Bueno, Giulia Mizzon, Jason J. Davis; Capacitance spectroscopy: A versatile approach to resolving the redox density of states and kinetics in redox-active self-assembled monolayers, The Journal of Physical Chemistry B). Copyright (2012) American Chemical Society. ${ }^{26}$ 


\section{THEORETICAL BACKGROUND}

Ionic capacitive phenomena

The electrochemical capacitance $\left(C_{\bar{\mu}}\right)$ encompasses ${ }^{40,41}$ not only geometrical electrostatic capacitance $\left(C_{e}\right)$ but also non-faradaic phenomena generally including, for instance, those arising from the double layer formed on exposure of electrode interfaces to a (redox) inert electrolytic solution. The simplest representation of such interfaces considers a term $\kappa$ as the inverse Debye length $\left(L_{D}=1 / \kappa\right)$ such as

$$
\kappa^{2}=\frac{2(z e)^{2} N_{0}}{\varepsilon_{r} \varepsilon_{0} k_{B} T^{\prime}}
$$

wherein $z$ is the valence number of the ions, $e$ is the elementary charge, $\varepsilon_{r}$ is the relative permittivity of the dielectric environment, $\varepsilon_{0}$ is the dielectric permittivity of the vacuum $\left(\sim 8.85 \times 10^{-12} \mathrm{~F} \mathrm{~m}^{-1}\right), \varepsilon_{r}$ is the relative permittivity, $k_{B}$ is the Boltzmann constant and $T$ is the absolute temperature and finally $N_{0}$ the ionic concentration in the interface (the "static" concentration of ions physically adsorbed at the surface of electrode). The associated interfacial ionic capacitance $\left(C_{i}\right)$ per unit area is ${ }^{29}$

$$
C_{i}=\varepsilon_{r} \varepsilon_{0} \kappa
$$

A more universal model arises from a consideration of both quantic $\left(C_{q}\right)$ and classical $\left(C_{e}\right)$ contributions to electrochemical capacitance phenomenon $\left(C_{\bar{\mu}}\right)$ as $^{42,43}$

$$
\frac{1}{C_{\bar{\mu}}}=\frac{1}{C_{e}}+\frac{1}{C_{q}}
$$

wherein $C_{e}$ has its classical electrostatic meaning, i.e. it is a constant that depends only on the dielectric properties of the environment and geometric factors. For a simple plate capacitor $C_{e}=\varepsilon_{r} \varepsilon_{0} \delta$, where $\delta=$ $1 / L$ and $L$ the distance between the plates. One can note here the similarity of this picture to the phenomenology presented by Eqn. (2), with the exception that $\delta$ is not ionic strength dependent (where $\kappa$ is). The physical origin of differentiation between electrostatic and ionic plate capacitors is, thus, contained within the meaning of the $\delta$ and $\kappa$ inverse length terms (see Figure 2). 
a)

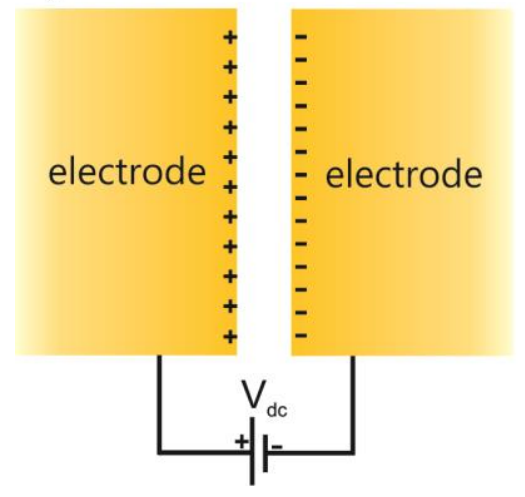

b)

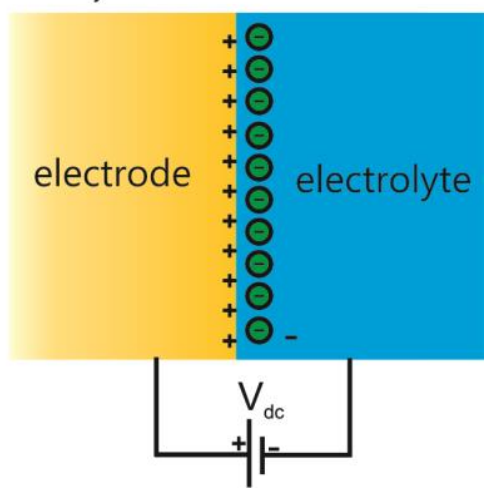

Figure 2. (a) Electrostatic plate capacitor in which the capacitance per unit of area is inversely dependent on the distance between the plates, $\delta=1 / L$, and (b) electrochemical plate capacitance wherein the capacitance is dependent on the inverse of Debye length, the $\kappa$ term; $\kappa$ depends on the ionic concentration and this is accordingly modified when a dielectric molecular film intervenes between the ionic layer in the electrolyte phase and the electrode (the metallic phase). We show here that molecular films additionally contain an ionic fingerprint that is herein referred as ionic relaxation.

$C_{q}$ is defined as ${ }^{15,40,42} 1 / C_{q}(\bar{\mu})=q / e^{2}\left[1 / g_{l}(\bar{\mu})+1 / g_{r}(\bar{\mu})\right]$, where $q=e N$ is the amount of electronic charge exchanged between the two different "plates"; $g_{\mathrm{l}}(\bar{\mu})=\mathrm{d} N_{\mathrm{l}} / \mathrm{d} \bar{\mu}$ and $g_{\mathrm{r}}(\bar{\mu})=\mathrm{d} N_{\mathrm{r}} / \mathrm{d} \bar{\mu}$ are the densityof-states of the "left" and "right" plates respectively (Figure $2 a$ ). Note that $\bar{\mu}$ refers to the energy state associated with the electrochemical potential of the electrons in the plates $(\bar{\mu}=\mathrm{eV}$, wherein $V$ is the potential of the electrode). If the two plates are metallic ideal conductors (as is the case of classical plate capacitor) the term $1 / C_{q}$ vanishes in the Eqn. (3), leaving $C_{e}$ as a good physical approximation of a more general $C_{\bar{\mu}}$. In the specific case of electro-active monolayers on metal (Figure 1 ) we have shown that $C_{\bar{\mu}}=$ $e^{2} \mathrm{~d} N_{\mathrm{r}} / \mathrm{d} \bar{\mu}_{1}^{15,26}$ which is dominated by $C_{q}$. One can see, thus, that $C_{e}$ and thus $C_{i}$ represent poor approximations for $C_{\bar{\mu}}$ in the presence of an addressable redox system. For non-faradaic phenomena (Figure 1), Eqn. (3) becomes $1 / C_{\bar{\mu}}=1 / C_{i}+1 / C_{q}$ and, because $1 / C_{q}$ vanishes, the resultant measured electrochemical capacitance is $C_{i}$. $C_{i}$ is, then, a good physical approximation of non-faradaic relaxation only. The simplest way to demonstrate this is to appeal to the Thomas-Fermi model, ${ }^{29}$ a straightforward approximation of Lindhard's formulation of the electric field screening. ${ }^{44}$ In this model the wave vector, $\kappa$, previously associated to the inverse of Debye length, is given by $\kappa^{2}=\left[(z e)^{2} \mathrm{~d} N_{\mathrm{r}} / \mathrm{d} \mu\right] / \varepsilon_{\mathrm{r}} \varepsilon_{0}$, wherein it can be recognized that

$$
C_{\bar{\mu}}=\varepsilon_{\mathrm{r}} \varepsilon_{0} \kappa^{2}
$$

There are two main considerations to be made here. Firstly it contains a quantum mechanics connotation wherein the charging sites behave as non-classical states. That is, the occupying states are indistinguishable and physically different to those considered in Eqn. (2). Secondly, the capacitance depends on the square of 
the $\kappa$, a relationship born out of the indistinguishable nature of electrons. In the specific case of ionic (distinguishable particles) contributions to electric field screening, the classical approximation can be applied, such that $\mathrm{d} N_{\mathrm{r}} / \mathrm{d} \mu=2 N_{0} / k_{B} T$ and, therefore, $\kappa^{2}=\left[(z e)^{2} 2 N_{0} / k_{B} T\right] / \varepsilon_{\mathrm{r}} \varepsilon_{0}$. The Debye-Hückel model is thus confirmed to be a particular example of Tomas-Fermi predicting screening ${ }^{41}$ and Eqn. (2) a particular application of Eqn. (4), wherein $C_{i}$ is shown as a particular approximation of $C_{\bar{\mu}}$.

In summary, ionic capacitive phenomena (in a plate-like representation as exemplified in Figure $2 \mathrm{~b}$ ) are a common physical manifestation of a more general quantum mechanical screening ${ }^{42,43}$ occurring specifically at electrochemical interfaces. The CS resolved relaxation peaks (Figure 1) thus have the same origin, with faradaic relaxation being electron-dependent ${ }^{8,}$ 15, 42, 43 and non-faradaic (ionic) relaxation viewed from a classical mechanics perspective. ${ }^{41}$ With film modified electrodes, but in the absence of solution or surface confined redox processes, the latter ionic charging process is dominant but contains markedly more information than typically assumed within a "double layer" picture. Herein, we exam this in more detail, commencing with a consideration of realistic equivalent circuit representations.

a)

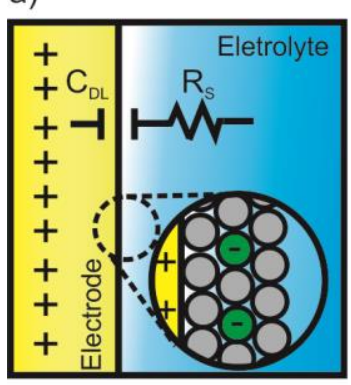

b)

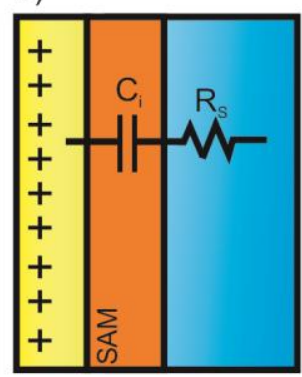

c)

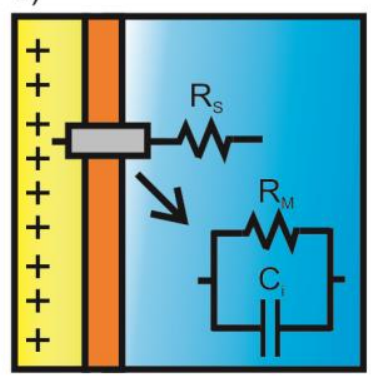

d)

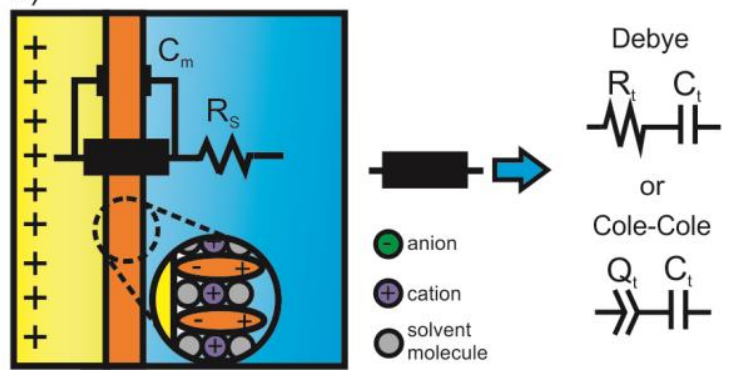

Figure 3. Equivalent circuit representations of (a) a bare electrode exposed to an electrochemically inert electrolyte solution. Within this, a rigid layer comprising solvent molecules and solvated ions exist and the ionic capacitance is generalised as $\boldsymbol{C}_{\boldsymbol{D} \boldsymbol{L}}$ (double layer capacitance). (b) The SAM formation disrupts this capacitive feature in generating a new combined capacitance term, referring here as $\boldsymbol{C}_{\boldsymbol{i}}$. (c) An interfacial configuration wherein the monolayer presents a $\boldsymbol{C}_{\boldsymbol{i}}$ capacitance and a monolayer resistance $\boldsymbol{R}_{\boldsymbol{M}}$ that physically represents field induced ionic translation within the film (rather than $\boldsymbol{R}_{\boldsymbol{t}}$ which is associated with the Cole-Cole rotational ionic relaxation). Note that the capacitances can be considered as a non-ideal capacitive elements, modelled as a CPE (constant phase element) defined as $\left[\boldsymbol{Q}(\boldsymbol{j} \boldsymbol{\omega})^{\boldsymbol{\alpha}}\right]^{-\mathbf{1}}$ where $\boldsymbol{j}=\sqrt{\mathbf{- 1}}$. (d) A more realistic model takes into account the dipolar/ionic relaxation and the ion/solvent ingress/trapping whose features can be described by the Debye (ideal situation) or Cole-Cole equivalent circuit. 


\section{Equivalent circuit models for ionic relaxation}

In terms of an associated equivalent circuit for a metal-solution interface, the ionic capacitance, $C_{i}$ (or $C_{D L}$ as its specific case), combines in series with the solution resistance $\left(R_{S}\right)$ (Figure $\left.3 a\right)$. A modification of the metal interface with a molecular layer significantly perturbs this ionic capacitance (Figure $3 b$ ), in a manner which has been previously detailed. ${ }^{27}$ Two circuit models have been applied at this point (Figure 3 ) in the absence of a redox probe in solution. The first (Figure $3 b$ ) describes the electrochemical response as resulting from a series combination of solution resistance, $R_{S}$ and a SAM capacitor, $C_{i}$, the latter usually replaced by a constant phase element (CPE) to take into account its non-ideal/frequency dependent form and dispersion, defined as $\left[Q(j \omega)^{\alpha}\right]^{-1}$ with $\alpha \sim 1$ and $j=\sqrt{-1}$ (Figure 3). ${ }^{23}$ Perfect dielectric and associated geometric capacitances are solely geometry $(\delta)$ dependent, in a manner different to ionic plate capacitance which has an intrinsic physico-chemical $(\kappa)$ dependence; the use of an ideal/geometric capacitance as circuit element should be strictly limited to situations where the SAM behaves unrealistically as a perfect dielectric, otherwise the dispersion is more appropriately modelled by the CPE and a physical interpretation for this should be appropriately assigned. The ionic insulator characteristics of a SAM are reportable by the phase angle in an electrochemical impedance analysis (where anything close to $-90^{\circ}$ at intermediate frequencies is typically taken as evidence of idealised double-layer capacitive behaviour). ${ }^{23}$ This idealised picture is not often observed in practice, ignores the leaky capacitive effects (which contribute significantly at low analytical frequencies where field induced motion can lead to ionic movement and energy loss within films; see Figure 5) and is, at best, physically incomplete. Recent studies have suggested that these SAMs behave as leaky capacitors, in the sense that there is a potential dependent ionic permeability, ${ }^{45}$ an associated ionic relaxation (with an $R C$-time constant, Figure $3 d$ ) and a low frequency field induced translational mobility represented by a resistance term $R_{M}$ (Figure 3c). ${ }^{25,31}$ The ionic relaxation characteristics are nicely resolved in CS spectra at intermediate frequencies and an observed "semi-circle"; see Figures 1 and $5 b$.

The primary purpose of the present work is to address the physical origin of this $R C$-time constant by understanding how its capacitive and resistive elements, the film charging fingerprint, are influenced by film structural characteristics. We demonstrate that ionic entrapment, within even highly crystalline films, is energetically viable and, once entrapped, ions remain within the film (responding capacitive-like) unless the interface is sampled at low AC frequencies; the latter represents an induced "ionic leakage" 25 resolved in CS spectra (in both phase angle and Nyquist capacitive plots) but is not of focus here.

To begin, we initially look at the impact of molecular film formation on "native" double layer ionic capacitance. The model (Figure $3 d$ ) first proposes a modification of $C_{D L}$ in generating a new capacitive term, $C_{m}$, according to (Eqn. 5):

$$
\frac{1}{C_{m}}=\frac{1}{C_{D L}}+\frac{1}{C_{b}}
$$


Here $C_{b}$ is the capacitance of the molecular "film" structure "without" ionic ingress i.e. only the bulk capacitance of the monolayer at static conditions. This model is then further modified by a consideration of ions entrapped within the film; as noted above these generate ionic relaxation features which can be quantified by two terms in series combination, $C_{t}$ and $R_{t}$ (Figure $3 d$ ). ${ }^{27}$ Here $C_{t}$ refers to the ionic capacitance which is intrinsically associated with $R_{t}$ to provide a fingerprint of the monolayer ionic relaxation. The $R_{t} C_{t}$ time constant responds (in a time- or frequency- dependent manner) primarily at intermediate frequencies (see Figure $5 a$ and $5 b$ ). ${ }^{27}$ The term $R_{t}$ represents, then, the resistance associated with rotational voltage perturbations within the film. $R_{M}$ is translational and is present at lower frequencies. $R_{t}$, our focus here, is calculated using an appropriate equivalent circuit (Figure $3 d$ ) or easily from the film non-faradaic relaxation time constant $\left(\tau_{t}\right.$, obtained as the inverse of the relaxation frequency of the film associated with non-faradaic phenomenon) since $R_{t}=\tau_{t} / C_{t} \cdot{ }^{27}$

It is important to note that while the model incorporates an idealized Debye description for the relaxation, a more plausible (Cole-Cole) description that considers a Gaussian distribution of ionic relaxation times and orientation energies in response to the alternating electric field is also contemplated. It only requires replacement of the resistive term with a non-ideal element ( $C P E$, for instance, as shown in Figure $3 d$ ) to account for a distribution of time constant responses. In either case, the complex capacitance response as a function of frequency $\left[C^{*}(\omega)\right]$ is described by (Eqn. 6): ${ }^{27}$

$$
C^{*}(\omega)=C_{m}+\frac{C_{t}-C_{m}}{1+\left(j \omega \tau_{t}\right)^{1-\beta}}
$$

where $j=\sqrt{-1}, \tau_{t}=R_{t} C_{t}$ and by considering $\alpha=1-\beta$ the ideal Debye phenomenon is restated by setting $\beta$ as null. Note that the equivalent circuit (Figure $3 d$ ) contained in Eqn. (6) can be described as $C_{m}$ (a capacitance charging at higher frequencies) in parallel with the series combination of $C_{t}$ (a capacitance predominantly present at intermediate to lower frequencies) and $R_{t}$, Figure $3 d^{27}$. It is worth noting that this circuit provides a more complete picture of the SAM modified surface than is normally considered because it contemplates the perturbed, solution phase, ionic distribution and the ionic relaxation response associated with entrapped ions at the interface. An ideal, defect-free, perfect dielectric SAM would, of course, present no $R_{t}, C_{t}$ or $R_{M}$. Real molecular films, are, however, rich with much more information (see Figures $4,5,6$ and early work). ${ }^{27}$ Here we analyse the model summarised in Figure $3 d$ and show how SAM crystallinity directly and predictably impacts resolved ionic film charging fingerprints. We additionally use molecular dynamics (MD) simulations to specifically identify the energetic barriers associated with ionic penetration. 


\section{EXPERIMENTAL PROCEDURES AND MOLECULAR SIMULATION}

\section{Experimental procedures}

All chemicals were purchased from Sigma-Aldrich, and all solvents from Fischer-Scientific, unless otherwise stated. Diamond polishing spray was purchased from Kemet. EIS experiments were performed on an Autolab Potentiostat 12 equipped with an FRA2 module, using gold electrodes (99.99\% purity, $1.6 \mathrm{~mm}$ diameter, sealed in a Teflon tube) purchased from BASi, platinum wire counter electrode and a mercury/mercury sulfate $\left(\mathrm{Hg} / \mathrm{HgSO}_{4}\right)$ reference electrode (BASi.). Ultra pure water was obtained from a Milli$\mathrm{Q}$ 18.2 $\mathrm{M} \Omega \mathrm{cm}$ system and was used in all solutions.

Polycrystalline gold electrode surfaces were prepared by mechanical polishing with diamond spray (0.1 micron). After sonication (five minutes) in water the gold electrodes were immersed in hot piranha solution (conc. $\mathrm{H}_{2} \mathrm{SO}_{4}: \mathrm{H}_{2} \mathrm{O}_{2}, v / v$ 3:1) for 5-10 minutes, before once again sonicating in water (five minutes). Finally electrodes were electrochemically polished in $0.5 \mathrm{M} \mathrm{KOH}$ between $-0.5 \mathrm{~V}$ and $-1.8 \mathrm{~V}$ followed by $0.1 \mathrm{M} \mathrm{H}_{2} \mathrm{SO}_{4}$ between $0 \mathrm{~V}$ and $1.25 \mathrm{~V}$ until a stable reduction peak is observed. The area of the gold electrode was determined from the gold/gold oxide reduction as reported previously. ${ }^{27}$

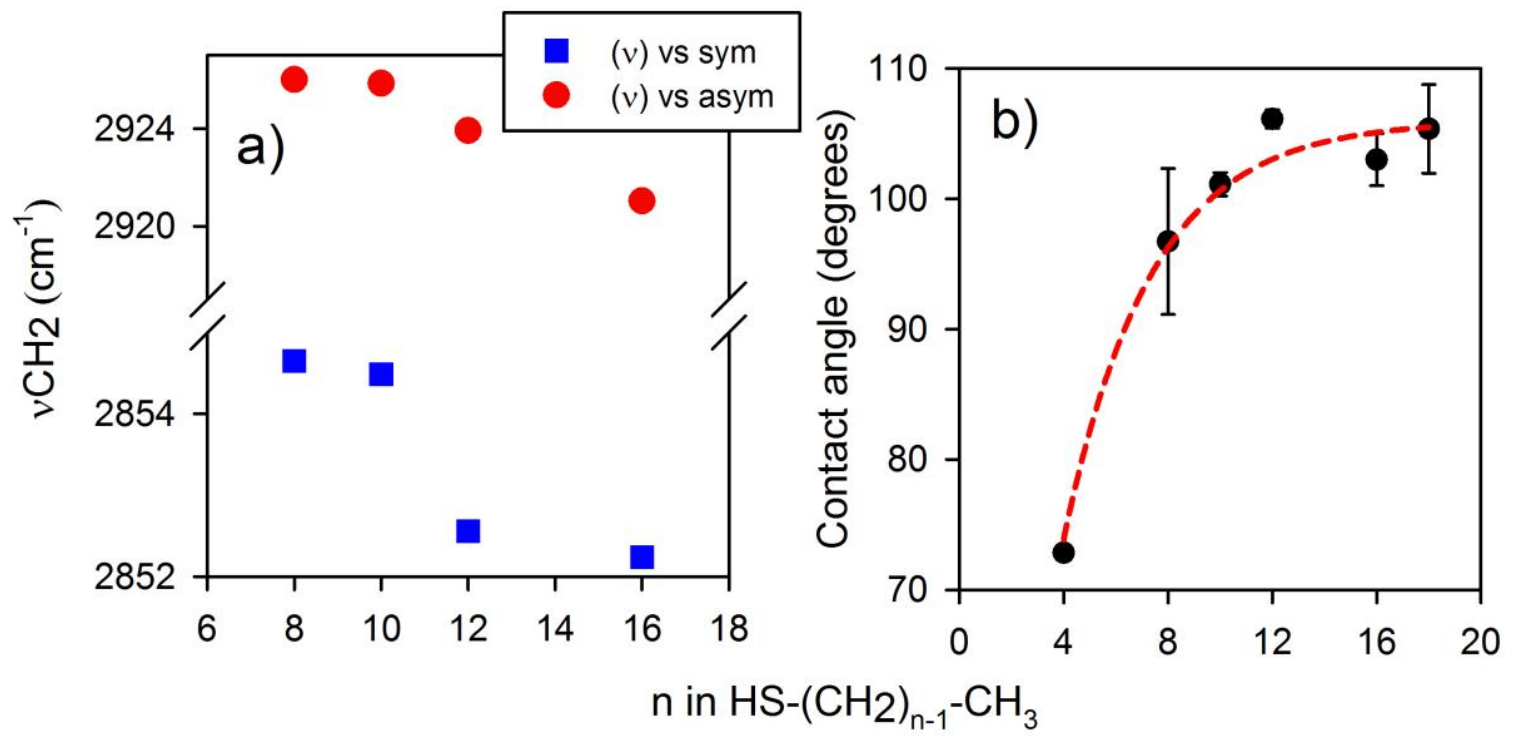

Figure 4. (a) Wavenumber of the asymmetric (red) and symmetric (blue) $\mathrm{CH}_{2}$ stretches versus alkanethiol chain length. (b) Water contact angle versus alkanethiol chain length.

Alkanethiol films formed from 1-butanethiol (C4), 1-octanethiol (C8), 1-decanethiol (C10), 1dodecanethiol (C12), 1-hexadecanethiol (C16) and 1-octadecanethiol (C18) were prepared by immersing the electrodes in $1 \mathrm{mM}$ thiol/ethanol solutions for $70 \mathrm{~h}$ (unless otherwise stated) $\left(25^{\circ} \mathrm{C}\right)$. After assembly gold electrodes were washed with copious amounts of ethanol followed by deionized water and electrolyte. 
Electrochemical measurements were carried out in $0.15 \mathrm{M}$ electrolyte/solvent. Impedance measurements were carried out between the frequencies of $1 \mathrm{MHz}$ and $10 \mathrm{~Hz}$ at open circuit potential. $Z^{\prime}$ and $Z^{\prime \prime}$ values were converted to $C^{\prime \prime}$ and $C^{\prime}$ using the equations $C^{\prime}(\omega)=Z^{\prime \prime} / \omega|\mathrm{Z}|^{2}$ and $C^{\prime \prime}(\omega)=Z^{\prime} / \omega|\mathrm{Z}|^{2}$. All experiments were measured three times and standard deviation error values calculated from these repeats. All experiments were also checked to satisfy the Kramers-Kronig relationship of time-invariance.
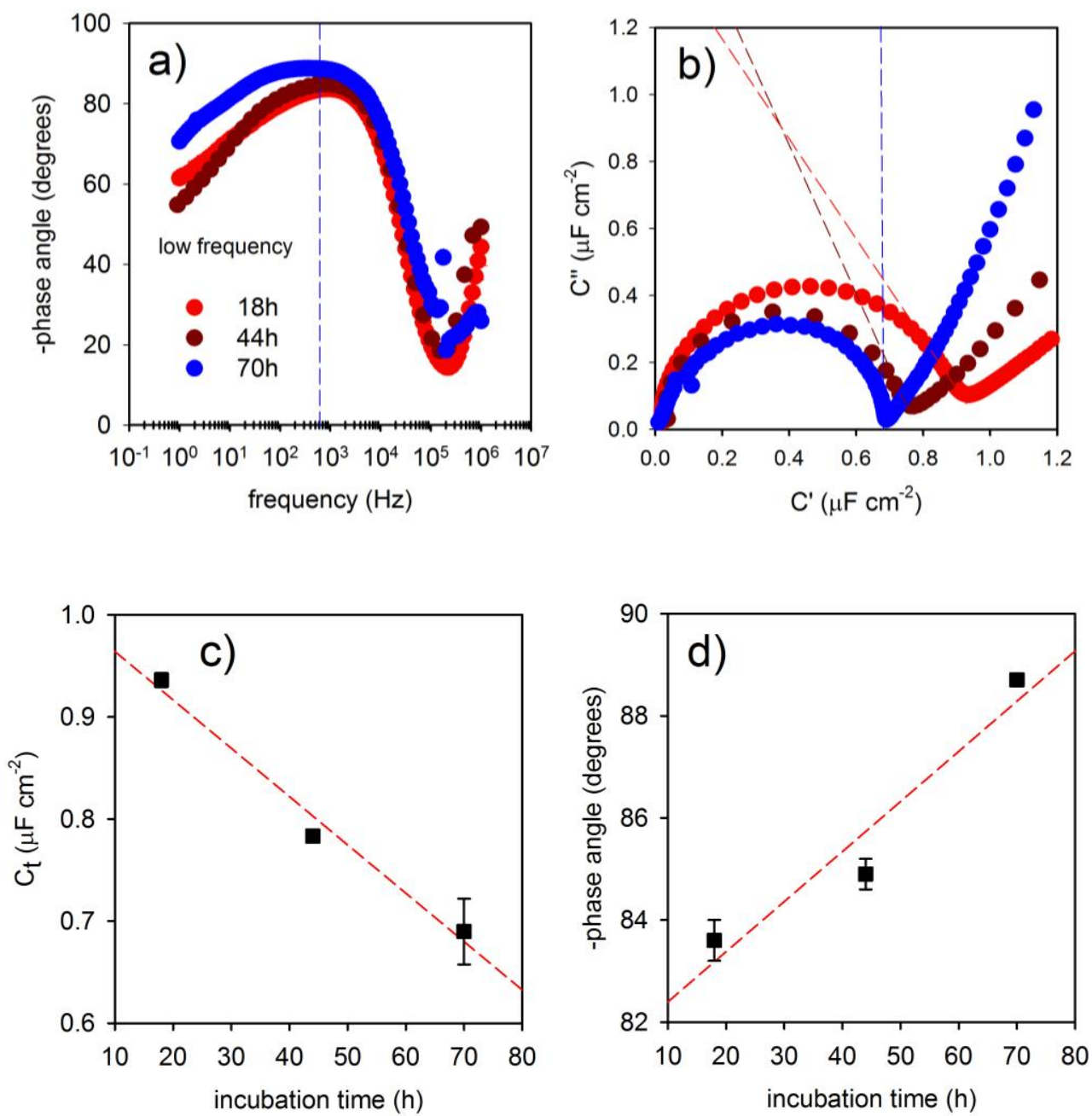

Figure 5. The influence of incubation time (in hours) on the phase angle [as shown in (a) and (d)] and on the interfacial ionic capacitance [shown in (b) and (c)]. It is evident that the phase angle, although close to $90^{\circ}\left(\sim 88^{\circ}\right)$ degrees at frequencies around $1 \mathrm{kHz}$ for $70 \mathrm{~h}$ of incubation times does not eliminate a resolved ionic relaxation (see also Figure 8 of reference ${ }^{27}$ ). In b) the deviation of the ideal capacitive behaviour is observed, in Nyquist diagrams, is highlighted by the deviation of the abscissa tangential slope from $90^{\circ}$ at the semicircle end (note a progression towards ideal for longer film incubations). In (c) and (d) the close correlation between $C_{t}$, incubation time and phase angle are reinforced. The vertical straight dashed line shown in (a) and (c) refers to the transition from intermediate to lower frequency. The semi-circlelike curves are, in all cases, attributed to the ionic relaxation process occurring inside the monolayers.

As in previous work ${ }^{27} C_{t}$ values can be calculated from the diameter of the semi-circle in a Nyquist plot of capacitance or by fitting the data using a Cole-Cole circuit. Peak frequency values were obtained from the maximum value in the Bode plot of $C^{\prime \prime}$. These are then converted to time-constants using $\tau_{t}=2 \pi / \omega$. 
In order to obtain a preliminary physical characterisation of alkanelthiol monolayers on gold, at various chain lengths, ATR-FTIR and water contact angle measurements were undertaken as shown in Figure 4. Gold substrates for ATR-FTIR were prepared by vapour deposition of $10 \mathrm{~nm}$ of chromium followed by $100 \mathrm{~nm}$ of gold onto a glass slide. Slides were immersed in piranha solution (conc. $\mathrm{H}_{2} \mathrm{SO} 4: \mathrm{H}_{2} \mathrm{O}_{2}$, v/v 3:1) for five seconds then washed with deionised water and ethanol, prior to immediate immersion in $1 \mathrm{mM}$ solutions of the alkanethiols for incubations larger than 48 hours. ATR-FTIR spectra were recorded on a Shimadzu IRTracer100 fitted with an ATR accessory. Atmospheric background scans were recorded taking an average of 65 scans with Happ-Genzel apodization. Sample data was collected and averaged over 65 scans using a resolution of $4 \mathrm{~cm}^{-1}$ for each measurement. Data was collected between 600 and $4500 \mathrm{~cm}^{-1}$.

Gold substrates for water contact angle measurements were prepared by vapour deposition of $10 \mathrm{~nm}$ of chromium followed by $100 \mathrm{~nm}$ of gold onto Si (100) substrates (Silicon Materials After cleaning with piranha solution ( 5 seconds) the substrate was immersed in a $1 \mathrm{mM}$ solution of the required alkanethiol for $48 \mathrm{~h}$. The gold substrate was then washed with deionised water and dried under nitrogen. Measurements undertaken by dispelling a $100 \mu \mathrm{L}$ on the surface and recording a photo using a CCD Camera. Complementary contact angle and ATR-FTIR data was acquired across all films (Figures 4 and 5).

\section{Molecular simulations}

The molecular dynamics (MD) structural model constructed to represent the gold-SAM interface is represented by a periodic metallic slab constituted by 300 gold atoms wherein the non-electroactive molecular layers are assembled over. The gold slab is arranged in 6 parallel layers of 50 atoms each. The configuration of the layers generates an $\mathrm{Au}(100)$ surface and an $\mathrm{Au}-\mathrm{Au}$ bond distance of $2.82 \AA$. On top of this metallic slab, the self-assembled monolayer is arranged by 25 alkanethiol molecules, organized in a planar structure with an angle of $30^{\circ}$ of inclination with respect to the direction perpendicular to the plane formed by the metallic slab. The sulfur atom from the alkyl group is deprotonated and covalently bonded to the gold surface, in the most stable configuration (as obtained from previous geometric computational optimization ${ }^{42}$ ) and kept fixed throughout the simulations. Hexanethiol and dodecanethiol were selected as being sufficient to demonstrate (within the computation time available) the presence of a thermally accessible SAM dependent energy barrier to ionic penetration into these SAMs. The gold-SAM MD structural model was treated using periodic boundary conditions, in a periodic cell with dimensions of $2.039 \times 8.223 \times 2.039 \mathrm{~nm}$. The cell contains explicit water molecules at the density of water at $1 \mathrm{~atm}$ and $300 \mathrm{~K}$, which yields approximately 750 water molecules. Only one positive ion is explicitly included in the centre of the cell, to represent a sodium ion crossing the monolayer. Relevant experimentally, the sodium cation was chosen to test the limiting condition of ionic permeation at low electrolyte concentration: although sodium is small, its charge density creates a significant hydration shell (the simulation results is in accordance with this), 
increasing its effective size. No other ion or counter ions were included. The resulting structural MD models are depicted in Figure 6 (as snapshot of initial molecular configuration of the electrode/SAM interface).
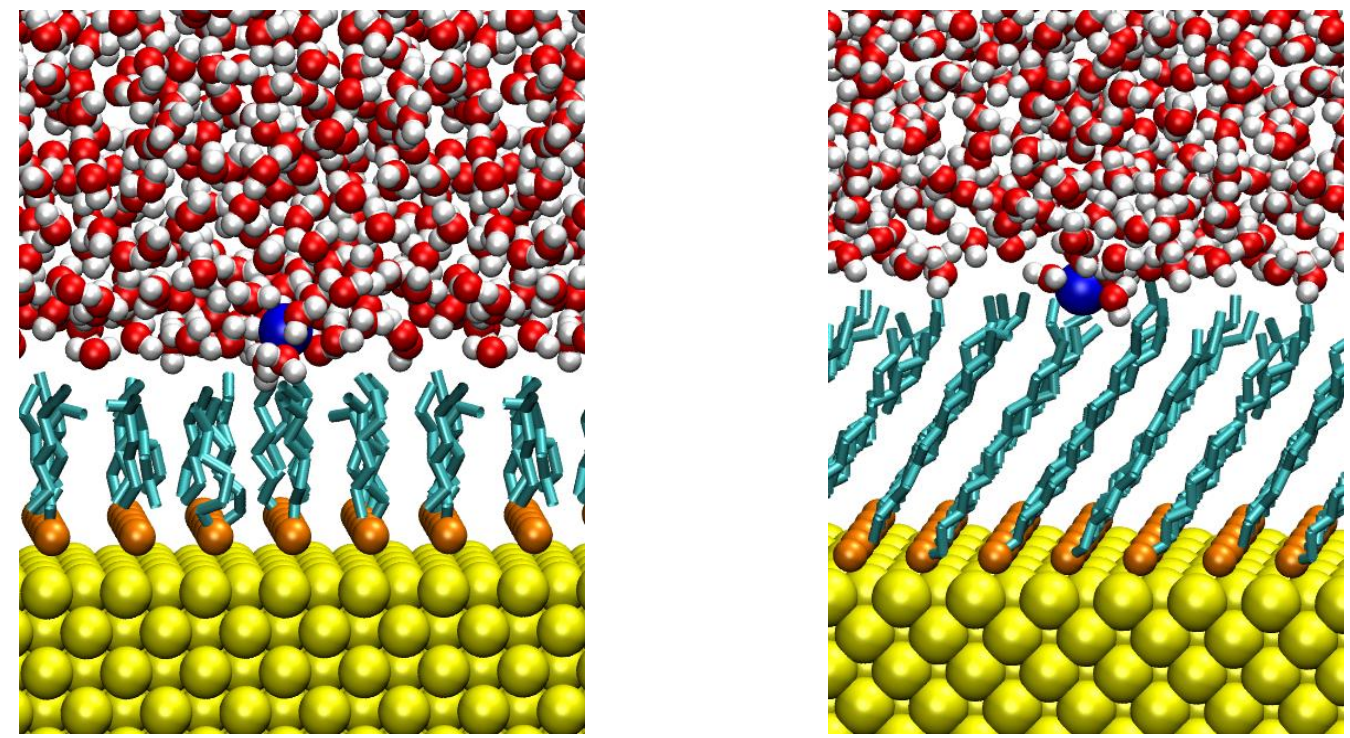

Figure 6. Atomistic illustrations of the SAM-hexanethiol-gold (left) and SAM-dodecanethiol-gold (right) junctions in water with an explicit sodium ion (blue sphere). The sulphur atoms are illustrated in orange in contact with the gold slab. This atomistic model was the starting point (evolving through the simulation) for the molecular structural configuration of the SAMs, under the appropriate (experimentally followed) conditions of the temperature and pressure, as well as the potential energy as defined by the force field parameterization.

The atomic system as shown in Figure 6 was submitted to a classical molecular dynamics (MD) simulation using the AMBER99SB ${ }^{46}$ force field parameterization implemented on the GROMACS package. ${ }^{47}$ The electrostatic interactions are treated using the Particle-Mesh-Ewald (PME) technique, ${ }^{48}$ which imposes that the system is periodic and neutral, with respect to the total charge. In order to include the effect of the electrode potential (to simulate the experimental conditions of $-0.42 \mathrm{~V} v \mathrm{SSCE}$ ), a uniform charge density were distributed among the gold atoms as estimated from experiments (see SI document). As the charge can be obtained from the experimental capacitance, a charge density can be experimentally inferred from the experiments. This charge density (that generate a potential consistent with experimental) was used to input in the MD simulation which is needed to mimic how the ions on the monolayer side (in contact with the electrolyte) will presumably evolve during the MD steps. The metallic side was accordingly charged at $4 \mu \mathrm{Ccm}^{-2}$ consistent previous theoretical studies. ${ }^{49,50}$

The water physical characteristics (in the electrolyte side of the junction) was selected by the SPC force field parameterization. ${ }^{51}$ At the same time, the total surface area corresponds to the minimum area for which the total surface charge is exactly compensated by the sodium ion charge $(+1 \mathrm{e})$, which results in a $-0.02 \mathrm{e}$. atom $^{-1}$ charge for the surface also in agreement to those attained experimentally. 
Under the described potential energy parameterization, an initial MD thermalization at $300 \mathrm{~K}$ for $100 \mathrm{ps}$ was carried out, using the Nosé-Hoover thermostat algorithm. ${ }^{52,53}$ The initial velocity of the atoms is assigned randomly, under a Maxwell-Boltzmann distribution, at the same temperature. In the following run, the system is subjected to a 200 ps constant pressure MD simulation under the pressure of $1 \mathrm{~atm}$, for the sake of system density equilibration, using the Parrinello-Rahman barostat algorithm. ${ }^{54}$ The pressure is applied only in the $y$ direction, in order to preserve the structure of the SAM-electrode system along the xz-plane of the surface. From the MD trajectories, a 2 ns non-equilibrium MD simulation was performed, by applying a time dependent harmonic potential on the sodium ion. The chosen coordinate for the potential was the distance between the sodium ion position and the position of the centre of mass of the electrode. The width of the potential biasing potential chosen for the sampling is the standard value of $1000 \mathrm{~kJ} \mathrm{~mol}^{-1} \mathrm{~nm}^{-1}$ and the rate of change of the equilibrium position $0.1 \mathrm{~nm} \mathrm{ps}^{-1}$. These initial configurations was allowed to float thereafter where the ion occupancy and structural possible positions where monitored with respect to the perpendicular y axis.

From this simulation, atomic configurations are selected for different sodium-electrode distances, ranging from 0.5 to $2.2 \mathrm{~nm}$, in a window of $0.1 \mathrm{~nm}$, yielding 18 initial frames. From each frame, a $50 \mathrm{~ns}$ NPT-ensemble umbrella sampling MD simulation ${ }^{50}$ at $300 \mathrm{~K}$ and $1 \mathrm{~atm}$ is made, for the sake of obtaining the average force on the sodium ion. The total sampling time for each monolayer type is $0.9 \mu \mathrm{s}$. From the average force, the potential of mean force (PMF) can be extracted, as the reversible work performed on the ion, and in constant pressure-constant temperature simulations, this corresponds to the Gibbs free energy, once the effect of the external potential is removed. This is done employing the weighted histogram analysis method (WHAM), ${ }^{55}$ where the free energy surface is obtained.

\section{RESULTS AND DISCUSSIONS}

\section{SAM crystallinity versus capacitance spectroscopic measurements}

As expected the asymmetric (red) and symmetric (blue) $\mathrm{CH}_{2}$ stretching frequencies were observed (Figure 3a) to shift from $2923 \mathrm{~cm}^{-1}$ to $2918 \mathrm{~cm}^{-1}$, and from $2855 \mathrm{~cm}^{-1}$ to $2851 \mathrm{~cm}^{-1}$, respectively as alkyl chain length increases from $n=10$ (decanethiol) to $n=18$ (octadecanethiol), highlighting an expected increase in crystallinity with chain length. ${ }^{56}$ Impedance analyses were conducted without the use of a redox probe in solution at open circuit potentials for all modified electrodes. An increase in impedance modulus is observed at frequencies below $10^{3} \mathrm{~Hz}$ (Figure $7 a$ ) an observation assigned to the lower frequency ionic leakage from films. To evaluate the capacitive and resistive contributions separately across the ionic relaxation frequency region of interest $\left(10-10^{6} \mathrm{~Hz}\right)$, the phase angle (i.e. the shift between the time-voltage perturbation and the time-current response to this perturbation) was recorded. The respective Bode plots (Figure 7b) reveal that, at high frequencies $\left(10^{6} \mathrm{~Hz}\right)$, the capacitive contribution $\left[C_{m}\right.$, following Eqn. (6)] is clearly resolved, until 
$\sim 10^{5} \mathrm{~Hz}$ where the phase angle is nearly null. At intermediate frequencies (between $10^{5}$ and $10^{3} \mathrm{~Hz}$ ) the $C_{t}$ capacitive element is predominant and phase is close to, but slightly lower than that expected for idealised defect-free SAMs (phase angle $\geq-88^{\circ}$ ); ${ }^{23}$ this may be indicative of the presence of defects that enable ionic permeation into the film (both during incubation and stabilization before measurement), regardless of film chain length. Previous studies have shown that alkanethiol film crystallinity and dielectric performance are sensitively dependent on the incubation time. Boubour and Lennox ${ }^{23}$ demonstrated, for example, that 1 undecanethiol films take up to $40 \mathrm{~h}$ to present phase angles exceeding $88^{\circ}$. Across the films utilised herein, we indeed reproduce this trend $\left(83^{\circ}\left[20 \mathrm{~h}\right.\right.$ ], to $\left.89^{\circ}[70 \mathrm{~h}]\right)$, and support it with FTIR observations (Figures 4 and 5). Indeed $\tau_{t}$ can be also precisely used as fingerprint of incubation time and crystallinity as shown in Figures 5 and $8 b$ (inset).

As noted in the introduction, film capacitive features are better evaluated by converting all impedance raw complex data $\left[Z^{*}(\omega)\right.$, Figure $\left.7 c\right]$ into complex capacitance $\left[C^{*}(\omega)\right]$ (Figure $\left.7 d\right)$, where $C_{t}$ values are readily resolved (from the semicircle diameter or fitting, using Eqn. (6), the data using Debye or Cole-Cole circuits, Figure $7 d$ ). ${ }^{27}$ The FTIR and phase resolved evolution ${ }^{57}$ of film crystallinity with time is also mapped out through $C_{t}$ trends (Figures $5 a, 5 d$ and Figure $7 b$ ). It is important to note that, for all of the films, impedimetric Nyquist plots (Figure 7c) exhibit a vertical line parallel to the y-axis over the frequency range studied (specially lower than $10^{3} \mathrm{~Hz}$ ). Higher than $10^{3} \mathrm{~Hz}$ these lines are not parallel which is assigned to the previously described leakage we are not modelling here. From a consideration of geometry alone and in concordance with Eqn. (2) or similarly an ionic plate capacitive model ${ }^{27}$ (as exemplified in Figure 2), resolved $C_{t}$ is expected (Figures $7 d$ and Figure $8 a$ ) to fall with increasing monolayer thickness (Figure $7 a$ ). As $C_{t}$ is an ionic capacitance, this is in accordance with both electric field screening $(\kappa)$ and a progressively higher potential barrier for ionic penetration for thicker SAMs. Calculated film dielectric constants [Eqn. (2)] were in general agreement with previous literature reports ${ }^{23,27}$; since here $C_{t} \gg C_{m}$, the relaxation time is $\tau=\left[\left(R_{S}+R_{t}\right) C_{t}\right]$ (see Figure 8 and legends therein) which coincides with $\tau=R_{S} C_{d l}$ as typically interpreted. ${ }^{23}$ In other words, although the dielectric constants obtained from $C_{d l}$ or $C_{t}$ are mathematically equivalent [according to Eqn. (2)], they represent different forms of $C_{i}$. $C_{t}$ itself cannot be experimentally dissociated from $R_{t}$ (the uncompensated monolayer resistance $)^{27}$ in the resolved time-constant $\left[\left(R_{S}+R_{t}\right) C_{t}\right]$. These physical features are unresolved in transient (chrono-type) or cyclic voltammetric experiments analyses of monolayer films. ${ }^{27,28}$ 
a)

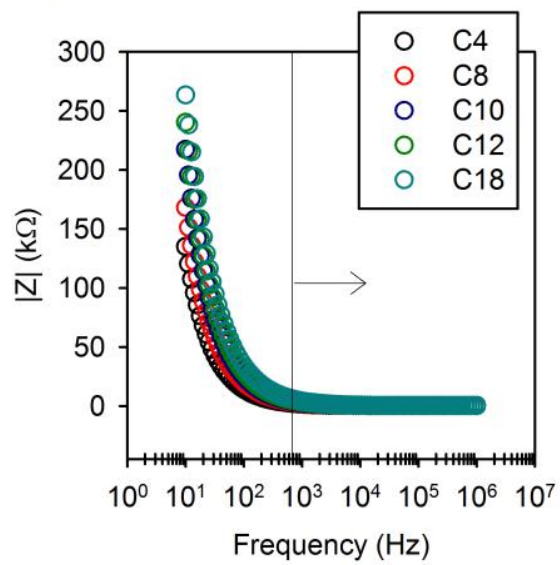

c)

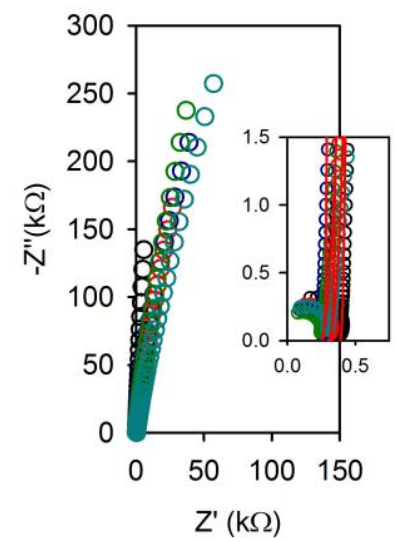

b)

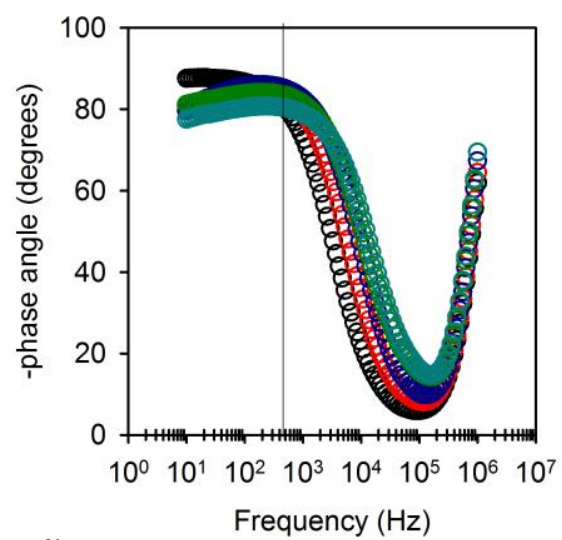

d)

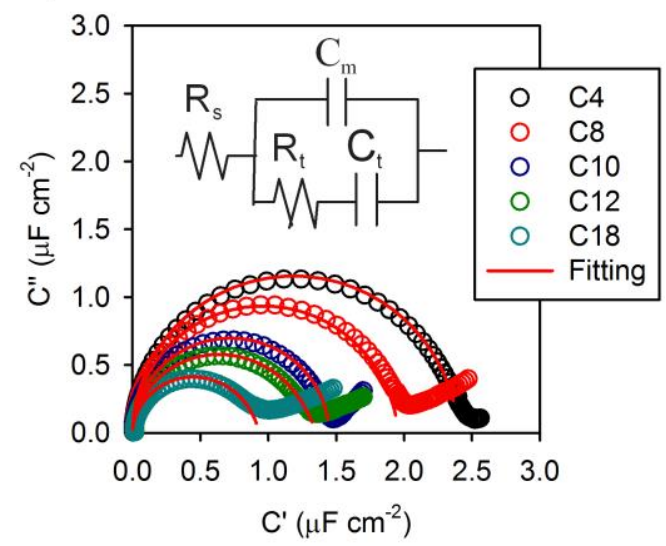

Figure 7. Bode modulus plots: (a) and Bode phase angle plot (b) acquired at OCP using $0.15 \mathrm{M} \mathrm{NaOCl}_{4} / \mathrm{H}_{2} \mathrm{O}$ for a range of alkanethiols. The vertical lines demonstrate the frequency region were the equivalent circuit used in (d) is committed. Impedance (c) and capacitance (d) Nyquist plots. Note that the inset in (c) represents the fitting plots (red lines) in all frequency range excluding the ionic diffusion which will not be discussed herein. Observe that from the Nyquist impedance plot, it is difficult to extract any information regarding capacitive and resistive contributions (the relaxation itself). On the other hand, converting the impedance raw complex data to complex capacitance, it is possible to obtain the capacitive behaviour of the interface SAM modified electrode/solution. The inset in (d) represents the equivalent circuit used to fit (red lines) the data, which represents (phenomenological model) essentially a Cole-Cole relaxation circuit as described in Eqn. (6) with $\alpha \sim 0.3$.
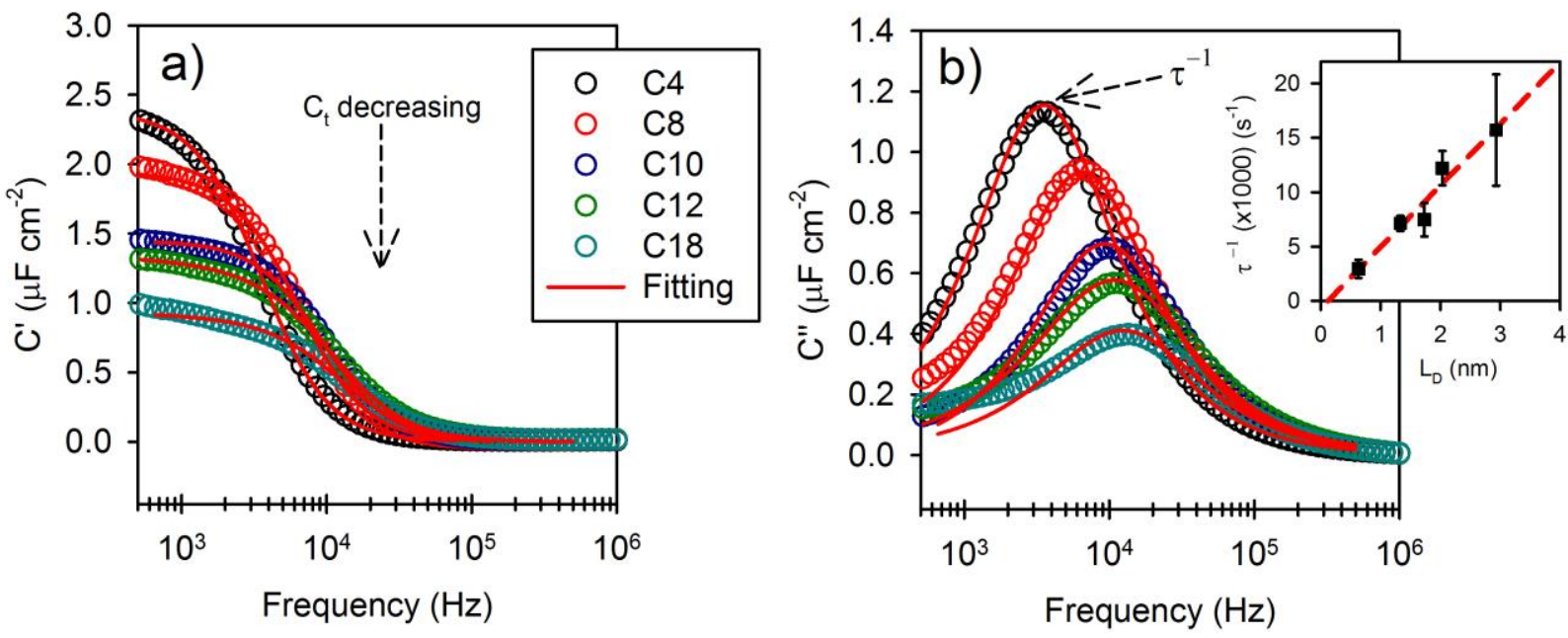
Figure 8. Bode diagrams of the (a) real and (b) imaginary parts of the complex capacitance for all films studied. In part (a), note that $C_{t}$ values (evaluated from plateau heights) decrease as $L_{D}$ (monolayer thickness) increases. Within the imaginary capacitance, it is possible to observe the dipolar-relaxation of the SAM, since the peak in $C^{\prime \prime}$ corresponds to the time constant $\tau^{-1}=\left[R C_{t}\right]^{-1}$, where $R$ is the series combination of solution resistance and monolayer resistance $\left(R=R_{s}+R_{t}\right)$. Note that $C_{m}$ value is minimal at high frequency and its contribution to the relaxation pattern is thus minimal. The red lines represent the fitting data using the Cole-Cole equivalent circuit model shown in the inset of Figure $6 d$. The inset demonstrates the linear dependence of $\tau^{-1}$ as a function of $L_{D}$ of the molecular layers as obtained from the analysis of $\tau^{-1}=\left[\left(R_{s}+R_{t}\right) C_{t}\right]^{-1}$.

Let us complement the previous analysis by noting that the imaginary Bode plot (Figure $8 b$ ) is a signature of Cole-Cole dispersion rather than ideal Debye behaviour (in a Cole-Cole Nyquist plot, the semicircle has a depressed format when compared with analogous Debye behaviour) ${ }^{27}$ in that it reports a dispersion of charging responses that can be estimated by $\alpha$. In this case, $R_{t}$ spans a distribution of values and is represented as a non-ideal element defined as $\left[Q_{t}(j \omega)^{\alpha}\right]^{-1}$ where $Q_{t}$ is a parameter. The distribution of the ionic relaxation times is quantified by $\alpha \sim 0.3$ for all measurements. It is worth noting that from imaginary Bode plots (Figure $8 b$ ), it is possible to analyse the time dependent relaxation process, $\tau_{t}$, and consequently estimate the resistive component of the SAM, $R_{t}$. Significantly, $R_{t}$ increases with SAM component chain length (Figure 9b), consistent with an increasing impediment to AC field induced ionic rotation as potential is progressively dropped. Film thickness and crystallinity features impact both $R_{t}$ and $C_{t}$ as noted in Figures 9 and $8 b$ and are thus resolved within the signature of $\tau=\left[\left(R_{s}+R_{t}\right) C_{t}\right]$.
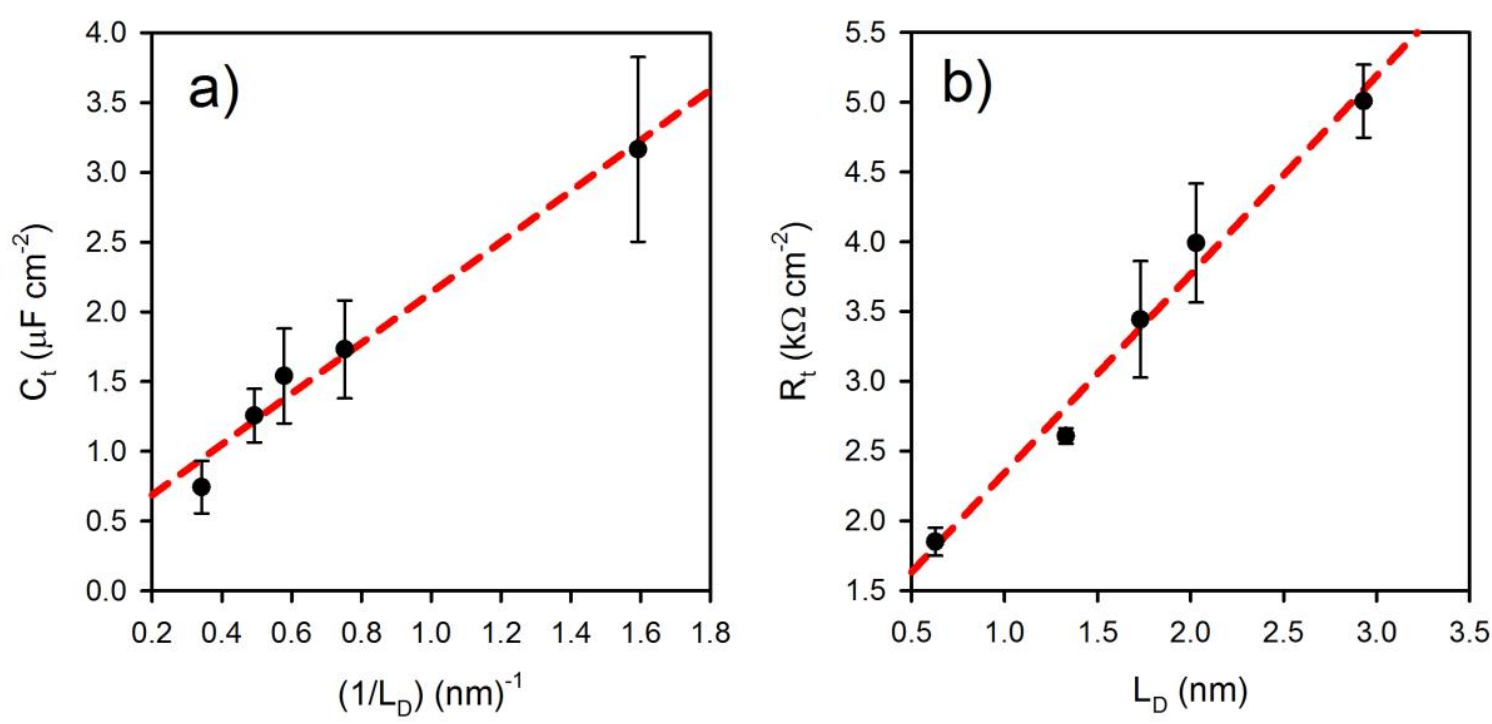

Figure 9. Evaluation of the element circuits by CS using the Cole-Cole relaxation phenomenology of Figure $1 d$. Capacitance $C_{t}$ in (a) and resistance of the monolayer $R_{t}$ in (b). Note that the capacitance $C_{t}$ increases as $\kappa=1 / L_{D}$ and as expected from a Helmholtz-type model as introduced in Eqn. (2). The resistance increases with SAM thickness, in concordance with the fact that longer chain alkanethiols form packed ordered SAMs structures than smaller thiols.

In summary, the proposed model establishes signatures of the ionic relaxation (through the $\tau_{t}$-time constant obtained by using CS) that serve as fingerprints of SAM crystallinity and associated ionic ingress (which can accordingly be chemically tuned). We demonstrate below that MD simulations support the 
imprinting of ionic character on these films wherein a thermally accessible barrier is presented but accessible under normal experimental conditions.

\section{Steered Molecular Dynamics calculations}

In order to analyse the energetic viability and structure imposed variance associated with ionic migration into these films, non-equilibrium MD calculations were employed. Molecular dynamics ${ }^{54}$ studies have previously been reported on solution permeation into monolayers, and a number of non-atomistic theoretical models have been proposed, ${ }^{56,57}$ in seeking to address deviations from idealised capacitance due to charge flux across the films.

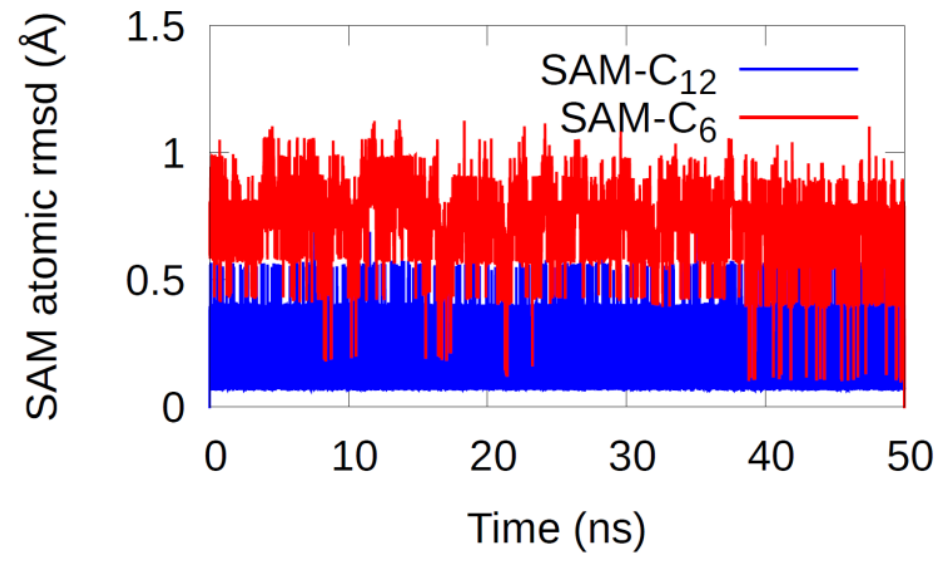

Figure 10. Root mean square deviation (RMSD) of the atomic positions of the alkanethiol carbon atoms from each studied SAM over a range of 50 ns MD simulation. The RMSD as obtained quantifies the thermal fluctuation of the atoms (of the atomic model considered) around average positions during the simulation. It thus confirms that atoms comprising the hexanethiol monolayer fluctuate (within the same kinetic energy) considerably more than the atoms within dodecanethiol along the simulation timespan.

Figure 10 illustrates the fluctuation of the atomic positions (along $x, y, z$ coordinates as explained in the legend) from each monolayer. It can be seen that the SAM-hexanethiol shows a higher positional fluctuation than is observed for dodecanethiol, an observation correlating with film mechanical rigidity / crystallinity. Ion permeation in these monolayers is also analysed by plotting pair-correlation functions, as shown in Figure 11. During ionic ingression into the monolayer, the water structure around the sodium ion changes in a manner dictated by the sterically tight packing within the model (a perfectly packed SAM system is assumed - Figures 6 and 11). This is seen as a decrease in the peak of the correlation function between the sodium atom and the water oxygens. The sodium-water attraction is very high and the first hydration shell enters the monolayer along with the ion. A closer look at longer distances of the distribution functions (quantifying the number density of oxygen atoms within a spherical shell of radii $r$ and $r+d r$, where $r$ is the current distance from the central atom of the distribution) reveals additional broad peaks at 0.4 and $0.6 \mathrm{~nm}$ (distance from the sodium ion; (see Figure 11). Thus, when the sodium atom enters the SAM, it drags a "unidirectional path" of organized water molecules with it (see Figure 13), generating an imprinted trajectory within the SAMs. This is 
seen in the MD trajectories as additional water molecules entering by the path created by the ion (Figure 11). ${ }^{54}$ These simulations also demonstrate that the solvent path imprinted in the SAMs structures is associated with a more organized water solvent structure then observed in the solvent bulk (see Figure 12).
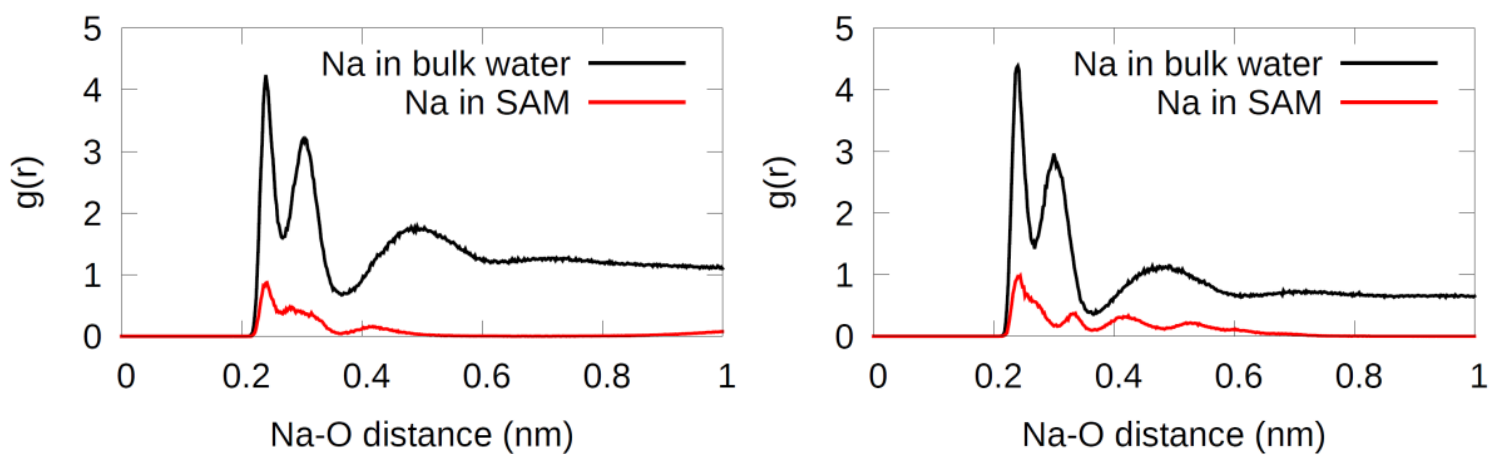

Figure 11. Radial pair distribution function $g(r)$ for the sodium - oxygen distance averaged in two different moments of the MD simulation, for the SAM-hexanethiol (left) and SAM-dodecanethiol (right). It can be observed that Na-O monitored in bulk water (black) is film independent. As times evolves Na penetrates into the SAM and the Na-O distance, monitored by $g(r)$ (red ) is initially $(0.2$ to $0.3 \mathrm{~nm}$ ) the same for both films? Then what?, but different in between 0.3 to $0.4 \mathrm{~nm}$.film penetration depth, or water-electrode distance or Na-O distance? From this it can be inferred that the penetration of $\mathrm{Na}$ in the monolayers is influenced by the film structure (and the environment solvent shown where?) predominantly for longer distance from the electrode. The area of the $g(r)$ curves can be used to infer about the ionic strength inside the monolayers, infer about the ionic strength? which is higher for thinner monolayers. This is reliable ???? with the ionic models inferred from Eqn. (2) as a physical approximation from Eqn. (4), so that consistent with an ionic electric field screening. ?????

The free energy profiles obtained from molecular dynamic simulations are analysed and shown in Figure 11 , and resolve the free energy changes associated with ionic movement into the film from solution. In both cases, the plateaus at great distances represent the situation where the energy does not vary appreciably, which corresponds to the situation close to the ion in bulk solution. The difference between the energy in this plateau and the highest energy represents the barrier to ion entrance into the monolayer. At low distances there is a second plateau corresponding to the ion being at the closest possible distance from the electrode surface. The difference between this energy and the highest energy corresponds to the barrier for ion loss from the electrode surface.

An potential biased MD simulation enables a calculation of the energy difference between the biased and unbiased (not electrically polarized) circumstances, building up a "potential of mean force" and an associated "resistance" and energetic barrier to ionic ingress. For the hexanethiol monolayer, the calculations resolve a penetration barrier of $\sim 3.8 \mathrm{kcal} \mathrm{mol}^{-1}$. This dehydration imparted electrically biased barrier is sufficiently small so as to suggest that a combination of SAM flexibility (Figure 10) and electrostatic forces are sufficient to enable its breaching. Furthermore, once the cation resides within the film it remains trapped and thereafter responsive to any imparted oscillatory field (unless the interface is moved to highly anodic potentials or 
perturbed for a longer time/lower frequency, as mentioned previously, wherein leakage occurs). For dodecanethiol, the energy profile shows interesting differences where two effects are noteworthy: (i) the corresponding SAM is not as conformationally labile so the intermolecular interactions are harder to overcome (ii) the ion enters the hydrophobic regions at a greater distance from the electrode and the cost for the removal of water is therefore higher. Specifically the penetration barrier has increased to $\sim 21.3 \mathrm{kcal} \mathrm{mol}^{-}$ 1. This higher barrier will essentially mean ionic penetration is markedly less probable. These calculated energy barriers (Figure 12) are consistent with previous theoretical studies, ${ }^{58,59}$ although their work differ in terms of PMF.
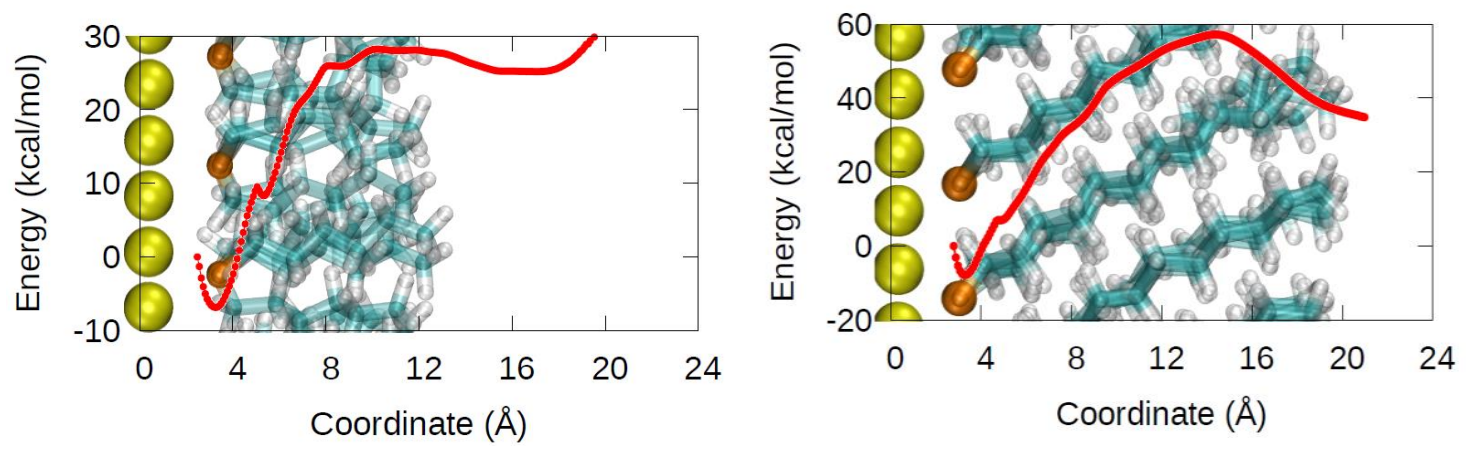

Figure 12. Free energy surface as a function of the sodium ion - gold surface distance coordinate, along with the corresponding SAM-metal illustration, with gold electrode surface atoms (yellow), sulphur (orange), carbon (cyan) and hydrogen (white) atoms.

These MD observations, although limited to just two experimentally relevant film thicknesses are selfconsistent with the $R_{t}$ trends as resolved by CS. We can directly associate resolved $R_{t}$ with the energy barriers to ionic ingress and a complete de-solvation of the second hydration shell, as demonstrated by radial distribution function (RDFs) plots (Figure 11); the sodium ion loses its second hydration shell almost completely but the first remains (Figure 12). With shorter chain length alkyls, the effects are two-fold: firstly, the electrostatic attraction of the electrode for the ion is, of course, greater. Secondly, by virtue of the less rigid molecular packing, the barrier to penetration is lower. 

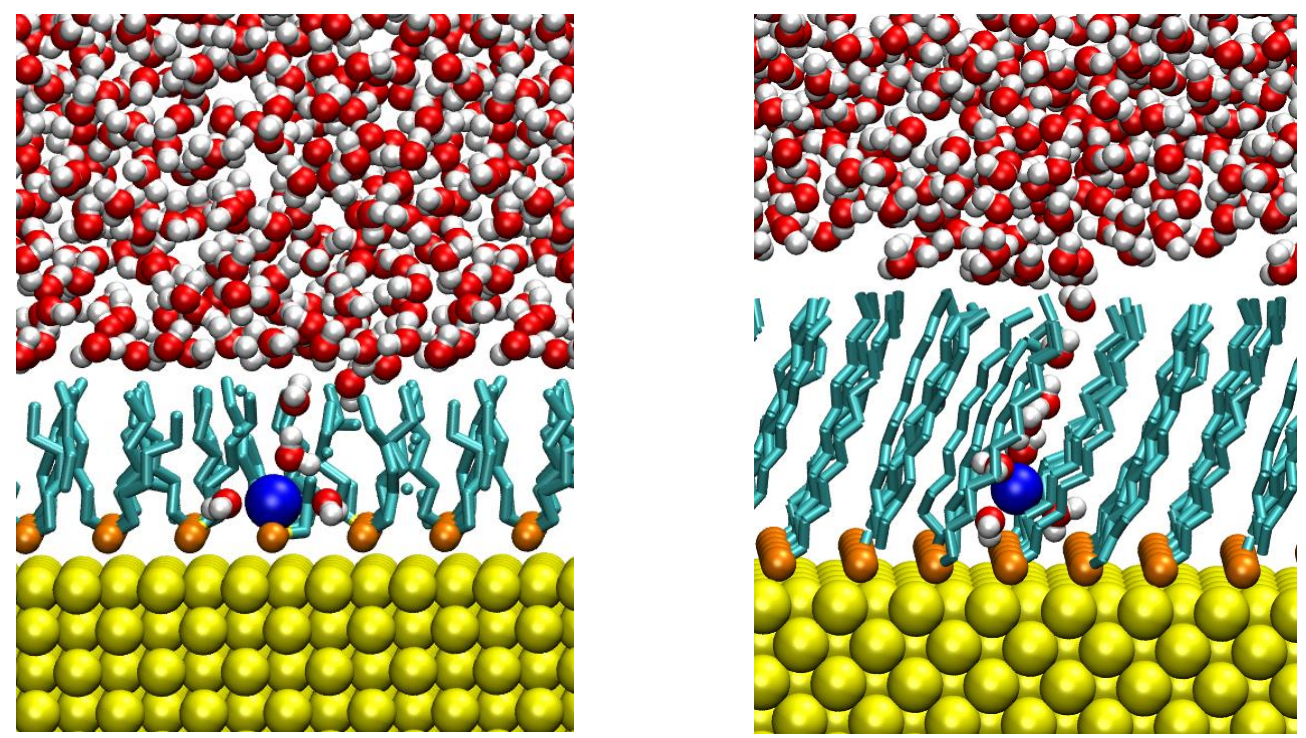

Figure 13. Atomistic illustration of an MD snapshot of the SAM-hexanethiol-gold and SAM-dodecanethiol-gold junctions in water with an explicit sodium ion (blue sphere), at the moment where the ion attraction for the electrode is maximum. These snapshots of the MD simulation, with the ion contained in the SAM, is a particular atomist evolution (in time) of the MD simulation as largely demonstrated by RDF plots: the snapshots visually depict a sodium ion entering the monolayer within a smaller hydration shell. The RDF graphs Figure 10 are the complete visualisation as they show what happens along the whole simulation time, but these snapshots are useful visual atomistic configuration to interpret the MD structural results.

\section{FINAL REMARKS AND CONCLUSIONS}

A simple double layer capacitance model is inadequate for a complete description of the charging and ionic resistive characteristics of the alkanethiol SAM modified electrode/solution interface. An organic layer introduced between an electrode and solution inherently decreases the interfacial capacitance but additionally introduces resolvable terms associated with both dipolar fluctuation and ionic relaxation due to ionic ingress and additional ionic contributions within the molecular film. These ionic characteristics are described herein using a range of alkanethiol film chain lengths with effects cleanly resolved by CS, without the use of a solution phase redox probe.

In terms of an equivalent circuit representation, the film modified interfaces were confirmed to be representable (across high to intermediate frequency regions) as a combination of capacitor and resistive terms. Contributions from film heterogeneity mean that these are in fact comprised of terms that respond differently as a function of an oscillatory frequency perturbation, such that Cole-Cole behaviour is predicted and observed. At higher frequencies, $C_{m}$ is predominant while at intermediate frequencies, $C_{t}$, the film ionic capacitance itself is observed to be present and dominant. These charging elements are clearly observed in the phase angle Bode diagram. In addition to the capacitive behaviour, the model comprises a (rotational) resistance $R_{t}$ in series with $C_{t}$, where $R_{t} C_{t}$ (resolved as $\tau_{t}$ ) represents a fingerprint of the SAM/electrolyte junction. Entrapped ions within the films are thus responsible for the resolved intermediate frequency $\left(1 / \tau_{t}\right)$ 
ionic relaxation. At lower analytical frequencies a field induced translational motion with an associated $R_{m}$ is resolved but not considered herein since it does not affect the ionic capacitance/resistance relaxation model described. Molecular simulations resolve a penetration barrier associated with (i) the loss of part of the hydration shell of the ion and (ii) the electrostatic forces responsible for the in-built potential barrier. Still uncomfortable about this - what "potential barrier" do we discuss? A complete loss of hydration was not observed in the MD simulation results, suggesting the molecular films are amenable to at least some level of solvent penetration. The ionic trail through the film is accompanied by the formation of highly organized water inside the monolayer, quite different to that found in the solvent bulk. Although these simulations do support a realistic and structure dependent ionic penetration, we cannot, of course, rule out entrapment occurring during film preparation rather than after it. We have not been able to remove these charging signatures by increasing alkanethiol purity, extensively cleaning glassware and/or adding scrubbing agents such as EDTA to solution during film preparation, or by eliminating the solution resistance from analyses (see also Figure 8 of reference ${ }^{27}$ ). These observations, and the fact that capacitance fingerprints scale so clearly with film crystallinity, are supportive of post-preparation ionic trapping and some level of solvent access to film from bulk. In terms of resistance analysis, ionic relaxation resistance $\left(R_{t}\right)$ increases with thicker films in a manner supported by IR mode frequency, contact angle and MD simulation resolved penetration barriers either in an entirely agreement with resolved $C_{t}$ change. The torsional resistance, translational resistance and capacitive charging features resolved by CS thus represent a much richer signature of simple molecular films than has been reported to date.

\section{REFERENCES}

1. J. C. Love, L. A. Estroff, J. K. Kriebel, R. G. Nuzzo and G. M. Whitesides, Chemical Reviews, 2005, 105, 11031170 .

2. N. K. Chaki and K. Vijayamohanan, Biosensors and Bioelectronics, 2002, 17, 1-12.

3. S. Ferretti, S. Paynter, D. A. Russell, K. E. Sapsford and D. J. Richardson, TrAC Trends in Analytical Chemistry, 2000, 19, 530-540.

4. $\quad$ S. Dong and J. Li, Bioelectrochemistry and bioenergetics, 1997, 42, 7-13.

5. T. Wink, S. Van Zuilen, A. Bult and W. Van Bennekom, Analyst, 1997, 122, 43R-50R.

6. F. C. B. Fernandes, A. Santos, D. C. Martins, M. S. Góes and P. R. Bueno, Biosensors and Bioelectronics, 2014, 57, 96-102.

7. C. P. Collier, E. W. Wong, M. Belohradsky, F. M. Raymo, J. F. Stoddart, P. J. Kuekes, R. S. Williams and J. R. Heath, Science, 1999, 285, 391-394.

8. P. R. Bueno, T. A. Benites and J. J. Davis, Scientific Reports, 2016, 6, 18400.

9. $\quad$ N. J. Tao, Nat Nano, 2006, 1, 173-181.

10. F. C. Bedatty Fernandes, A. V. Patil, P. R. Bueno and J. J. Davis, Analytical Chemistry, 2015, 87, 12137-12144.

11. S. M. Marques, A. Santos, L. M. Gonçalves, J. C. Sousa and P. R. Bueno, Electrochimica Acta, 2015, 182, 946952.

12. A. V. Patil, F. C. Bedatty Fernandes, P. R. Bueno and J. J. Davis, Analytical Chemistry, 2015, 87, 944-950.

13. A. Santos, J. P. Piccoli, N. A. Santos-Filho, E. M. Cilli and P. R. Bueno, Biosensors and Bioelectronics, 2015, 68, 281-287.

14. P. R. Bueno and J. J. Davis, Anal. Chem., 2014, 86, 1977-2004.

15. P. R. Bueno and J. J. Davis, Anal. Chem., 2014, 86, 1337-1341.

16. J. Lehr, F. C. B. Fernandes, P. R. Bueno and J. J. Davis, Analytical Chemistry, 2014, 86, 2559-2564. 
A. Santos, J. J. Davis and P. R. Bueno, Journal of Analytical \& Bioanalytical Techniques, 2014, 0, -. J. S. Daniels and N. Pourmand, Electroanalysis, 2007, 19, 1239-1257.

F. Lisdat and D. Schäfer, Analytical and Bioanalytical Chemistry, 2008, 391, 1555-1567.

A. L. Eckermann, D. J. Feld, J. A. Shaw and T. J. Meade, Coord Chem Rev, 2010, 254, 1769-1802.

E. Boubour and R. B. Lennox, Journal of Physical Chemistry B, 2000, 104, 9004-9010.

E. Boubour and R. B. Lennox, Langmuir, 2000, 16, 7464-7470.

E. Boubour and R. B. Lennox, Langmuir, 2000, 16, 4222-4228.

L. V. Protsailo and W. R. Fawcett, Langmuir, 2002, 18, 8933-8941.

B. O'Brien, H. Sahalov and P. C. Searson, Applied Physics Letters, $2010,97$.

P. R. Bueno, G. Mizzon and J. J. Davis, Journal of Physical Chemistry B, 2012, 116, 8822-8829.

M. S. Goes, H. Rahman, J. Ryall, J. J. Davis and P. R. Bueno, Langmuir, 2012, 28, 9689-9699.

P. R. Bueno, F. Fabregat-Santiago and J. J. Davis, Analytical Chemistry, 2013, 85, 411-417.

F. A. Gutierrez, F. C. B. Fernandes, G. A. Rivas and P. R. Bueno, Physical Chemistry Chemical Physics, 2017, accepted.

R. P. Janek, W. R. Fawcett and A. Ulman, Journal of Physical Chemistry B, 1997, 101, 8550-8558.

H. Sahalov, B. O'Brien, K. J. Stebe, K. Hristova and P. C. Searson, Langmuir, 2007, 23, 9681-9685.

M. Schweizer, M. Manolova and D. M. Kolb, Surface Science, 2008, 602, 3303-3307.

W. Wang, S. Zhang, P. Chinwangso, R. C. Advincula and T. R. Lee, Journal of Physical Chemistry C, 2009, 113, 3717-3725.

G. K. Olivier, D. Shin and J. Frechette, Journal of Electroanalytical Chemistry, 2010, 639, 50-58.

N. Darwish, P. K. Eggers, S. Ciampi, Y. Zhang, Y. J. Tong, S. Ye, M. N. Paddon-Row and J. J. Gooding, Electrochemistry Communications, 2011, 13, 387-390.

A. J. Bard and L. R. Faulkner, Electrochemical Methods: Fundamantals and Applications, Wiley, 2nd edn., 2001.

R. K. Mendes, R. S. Freire, C. P. Fonseca, S. Neves and L. T. Kubota, Journal of the Brazilian Chemical Society, 2004, 15, 849-855.

B. Liu, A. J. Bard, M. V. Mirkin and S. E. Creager, Journal of the American Chemical Society, 2004, 126, 14851492.

S.-J. Ding, B.-W. Chang, C.-C. Wu, M.-F. Lai and H.-C. Chang, Analytica Chimica Acta, 2005, 554, 43-51. D. A. Miranda and P. R. Bueno, Phys. Chem. Chem. Phys., 2016, 18, 25984-25992.

F. A. Gutierrez, F. A. B. Fernandes, G. A. Rivas and P. R. Bueno, Phys. Chem. Chem. Phys., 2017, 19, 67926806.

P. R. Bueno, G. T. Feliciano and J. J. Davis, Physical Chemistry Chemical Physics, 2015, 17, 9375-9382.

D. A. Miranda and P. R. Bueno, Physical Chemistry Chemical Physics, 2016, 18, 25984-25992.

J. Lindhard and K. D. Vidensk, Selsk. Mat. Fys. Medd., 1954, 28, 1-57.

N. Darwish, P. K. Eggers, S. Ciampi, Y. Zhang, Y. Tong, S. Ye, M. N. Paddon-Row and J. J. Gooding, Electrochemistry Communications, 2011, 13, 387-390.

V. a. A. R. a. O. A. a. S. B. a. R. A. a. S. C. Hornak, Proteins: Structure, Function, and Bioinformatics, 2006, $65,712--725$.

H. J. C. Berendsen, D. van der Spoel and R. van Drunen, Computer Physics Communications, 1995, 91, 43-56.

T. Darden, D. York and L. Pedersen, The Journal of Chemical Physics, 1993, 98, 10089.

I. Burgessa, M. Lia, S. L. Horswella, G. Szymanskia, J. Lipkowskia, S. Satijab and J. Majewski, Colloids and Surfaces B: Biointerfaces, 2015, 40, 117-122.

G. M. Torrie and J. P. Valleau, Journal of Computational Physics, 1977, 23, 187-199.

H. J. C. Berendsen, J. P. M. Postma, W. F. van Gunsteren and J. Hermans, in Intermolecular Forces: Proceedings of the Fourteenth Jerusalem Symposium on Quantum Chemistry and Biochemistry Held in Jerusalem, Israel, April 13-16, 1981, ed. B. Pullman, Springer Netherlands, Dordrecht, 1981, pp. 331-342.

S. Nosé, The Journal of Chemical Physics, 1984, 81, 511-519.

W. G. Hoover, Physical Review A, 1985, 31, 1695-1697.

M. Parrinello and A. Rahman, Journal of Applied Physics, 1981, 52, 7182-7190.

S. Kumar, J. M. Rosenberg, D. Bouzida, R. H. Swendsen and P. A. Kollman, Journal of Computational Chemistry, 1992, 13, 1011-1021.

M. D. Porter, T. B. Bright, D. L. Allara and C. E. D. Chidsey, Journal of the American Chemical Society, 1987, 109, 3559-3568.

C. D. Bain, E. B. Troughton, Y. T. Tao, J. Evall, G. M. Whitesides and R. G. Nuzzo, Journal of the American Chemical Society, 1989, 111, 321-335.

S. A. Kislenko, V. A. Nikitina and R. R. Nazmutdinov, High Energy Chemistry, 2015, 49, 341-346.

S. A. Kislenko, V. A. Nikitina and R. R. Nazmutdinov, Phys. Chem. Chem. Phys., 2015, 17, 31947-31955. 